\title{
Velocidade do som em metais pelo método do tempo de VOO
}

\author{
Speed of sound in metals by the time of flight method

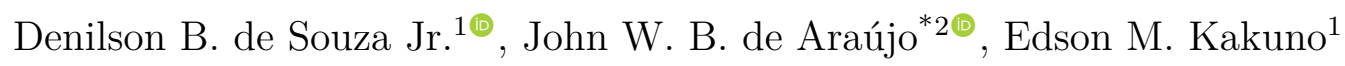 \\ ${ }^{1}$ Universidade Federal do Pampa, Campus Bagé, Bagé, RS, Brasil. \\ ${ }^{2}$ Universidade Federal do Rio Grande, Instituto de Matemática, Estatística e Física, Rio Grande, RS, Brasil.
}

Recebido em 28 de abril de 2020. Revisado em 28 de maio de 2020. Aceito em 01 de junho de 2020.

\begin{abstract}
Este trabalho propõe uma instrumentação, simples de manipular, destinado a aulas experimentais de ensino médio ou de graduação, capaz determinar a velocidade do som em barras metálicas através do tempo de voo, i.e., do tempo de propagação de um pulso longitudinal. O pulso é produzido a partir de uma perturbação (choque mecânico) em uma das extremidades de uma barra, sua velocidade é obtida através da razão do espaço percorrido pelo pulso e do tempo de propagação. Encontrou-se para o aço o valor de $4,7 \mathrm{~km} / \mathrm{s}$, com $5,3 \%$ de incerteza total em relação ao valor teórico.
\end{abstract}

Palavras-chave: Velocidade do som, Plataforma Arduino, Ensino de física, Ondas mecânicas.

\begin{abstract}
This work proposes a simple instrumentation, intended for experimental high school or undergraduate levels, capable of determining the speed of sound in metal bars through the flight time, i.e., the propagation time of a longitudinal pulse through the bar. The pulse is produced from a disturbance (mechanical shock) at one end of a bar, its speed is obtained by the ratio of the space traveled by the pulse and its propagation time. For steel, the value of $4.7 \mathrm{~km} / \mathrm{s}$ was found, with $5.3 \%$ of total uncertainty in relation to the theoretical value.
\end{abstract}

Keywords: Speed of sound, Arduino platform, Physics teaching, Mechanical waves.

\section{Introdução}

O método proposto é conveniente para aplicações em ensino, pois permite a interação do aluno, tanto para a execução do experimento como para a obtenção da velocidade. $\mathrm{O}$ experimento consiste em gerar uma perturbação em um ponto de uma barra e medir o tempo para esta perturbação alcançar um outro ponto da barra, e com o modelo de propagação da onda em meio uniforme, a velocidade é obtida, pela razão da distância entre os pontos e o tempo. Trabalhos que utilizam o mesmo princípio exposto podem ser vistos em $[1,2,3,4]$.

Este trabalho apresenta uma modificação da proposta de Speziali e Veas [1], no qual um pulso longitudinal, doravante pulso, é produzido em uma das extremidades de uma barra metálica, por exemplo, permitindo que esta sofra uma queda livre sobre uma superfície metálica rígida (denominada de base) e de massa muito maior do que a massa da barra. Quando a barra colide com a base, é produzido um pulso que se propaga na barra até a outra extremidade (livre) e como há uma mudança brusca de meio, extremidade livre da barra com o ar, uma parte significativa do pulso retorna pela barra, fazendo a mesma "pular" (ação e reação).

*Endereço de correspondência: johnwelvins@gmail.com
Durante o tempo do pulso percorrer a barra (tempo de voo), ela permanece em contato com a base, e quando o pulso retorna a extremidade na qual o choque foi gerado, através das forças de ação e reação a barra se separa da base, i.e., a base "empurra" a barra. Portanto medindose o tempo de contato da barra com a base, obtém-se o tempo para o pulso percorrer o comprimento da barra duas vezes (ida e volta) e calcula-se sua velocidade pela razão do dobro do comprimento com tempo, e esta corresponde a velocidade de propagação da onda sonora na barra. Speziali e Veas fazem uma analogia de uma perturbação se deslocando ao longo de uma mola suspensa na vertical.

O tempo de propagação do pulso na barra é pequeno e, portanto, também é pequeno o tempo de contato da barra com a base, inviabilizando sua medição de forma manual, por exemplo, com um cronômetro. Uma possibilidade nesta situação é a medida de alguma grandeza elétrica. O intervalo de tempo que se deseja medir encontra-se em torno de dezenas ou centenas de microssegundos, dependendo do comprimento da barra.

O contato entre a barra metálica e uma base, também metálica, funciona de forma análoga a uma chave do tipo liga-desliga. No trabalho de Speziali, essa "chave" é utilizada para descarregar um capacitor em um circuito série formado por um capacitor (C), um resistor (R) e a "chave", enquanto a "chave" estiver fechada o 
capacitor irá descarregar e o valor da carga resultante corresponde ao tempo do pulso se propagar por toda a barra e retornar.

Neste trabalho se propõe medir o tempo de contato entre a barra e a base utilizando um simples circuito eletrônico microprocessado, no caso com a plataforma Arduino.

Enquanto a "chave" está fechada o microprocessador fica contando o tempo, com resolução de microssegundos, e quando a "chave" abre, a contagem é interrompida e o valor do intervalo de tempo é registrado. O processo interno ao microprocessador é mais complexo que a descarga de um capacitor em um circuito RC, contudo a contagem do tempo é transparente ao usuário, tornando o experimento mais simples de ser realizado pelo estudante.

No experimento utilizando o circuito RC, o estudante deve compreender minimamente o processo de carga e descarga de um circuito RC. O tempo de contato é obtido de forma indireta, através da medição da tensão no capacitor, com um multímetro, sendo ainda necessário um cuidado extra de não deixar a barra bater na base duas ou mais vezes. Enquanto no método proposto o Arduino fornece diretamente o tempo em microssegundos e também registra somente o primeiro pulso, eliminando a interferência de múltiplos contatos da barra com base. Adicionalmente, caso se conheça previamente a geometria do experimento, i.e., o comprimento da barra, é possível aplicar um filtro de software que rejeite intervalos de tempo menores que um limite inferior predeterminado, por exemplo, que corresponda a metade do comprimento, e que um limite superior predeterminado, por exemplo, que corresponda ao dobro de comprimento, a aplicação deste filtro ajuda a eliminar erros de execução.

\section{Instrumentação}

O diagrama elétrico da instrumentação proposta é mostrado na Figura 1 e uma imagem da montagem física na Figura 2.

O Arduino aplica um potencial (nível lógico "1") no pino D12, o pino D10 permanece no nível "0", que é garantido pelo resistor R1, aguardando o nível " 1 " habilitado pelo pino D12, para iniciar a contagem de tempo. Quando a barra colide com a base, fecha o circuito elétrico e o nível "1" chega ao pino D10, iniciando a contagem de tempo, o término da contagem se dá no momento em que o nível " 1 " desaparece do pino D10.

O intervalo de tempo é registrado internamente no Arduino e este realiza a filtragem de software para determinar se o tempo está dentro do intervalo previsto e eliminar os pulsos seguintes. Passando pelo filtro, a coleta é contabilizada e o tempo é enviado ao computador pela porta USB, o valor do tempo é publicado em uma janela (monitor serial) de texto do computador.

Os materiais utilizados na construção foram: um Arduino, cabos para conexões, um segmento de trilho (base)

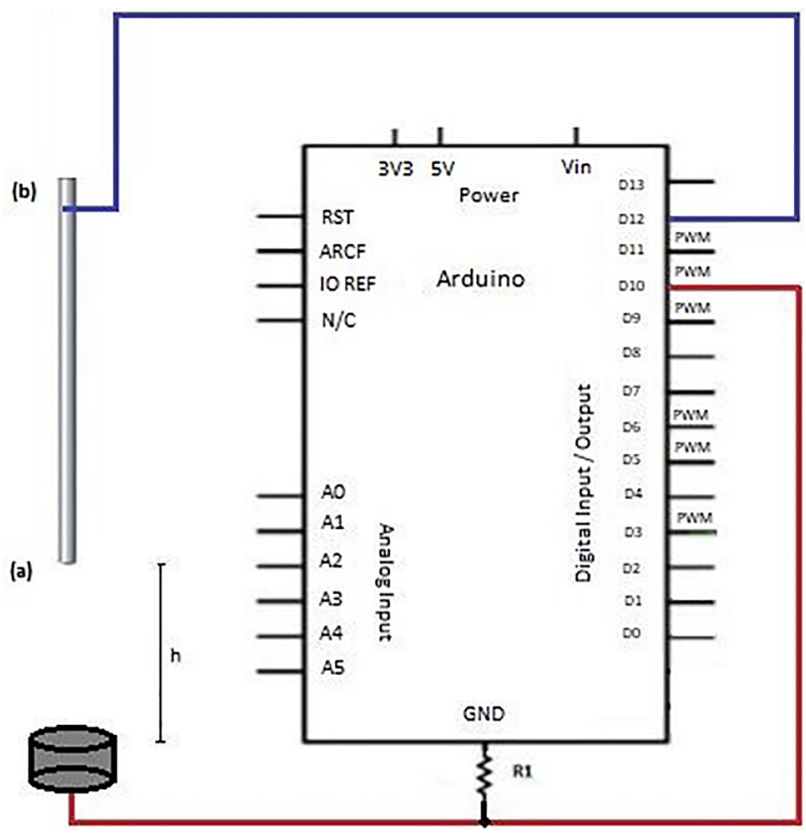

Figura 1: Circuito para captura do tempo de contato da barra com a base.

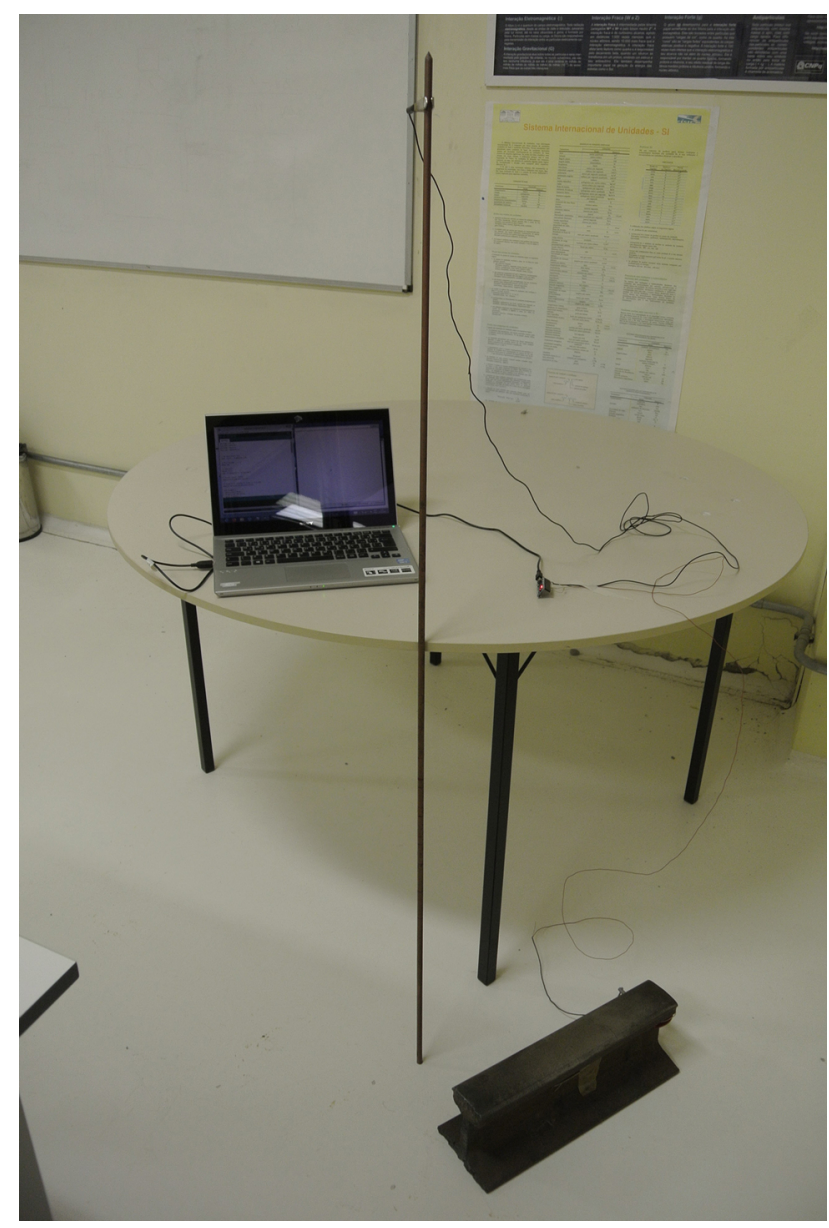

Figura 2: Imagem da montagem experimental. 
e uma haste de aterramento (barra), como mostrados na Figura 2.

Para coletar o tempo de contato da barra com a base, o programa mostrado no Código 1, utiliza a função pulseIn [5], esta função mede a largura temporal de um pulso na entrada digital selecionada (D10), seja ele "1" (HIGH) ou "0" $(L O W)$. A função pulseIn possui de dois a três parâmetros: o número do pino digital, pin, estado (" 1 " ou "0") em que o tempo será medido e o tempo máximo de espera (time out), que é opcional. No programa, Código 1, a opção time out não é utilizada, e caso seja, é

Código 1: Programa para determinação do tempo de propagação de um pulso para Arduino e outras plataformas compatíveis.

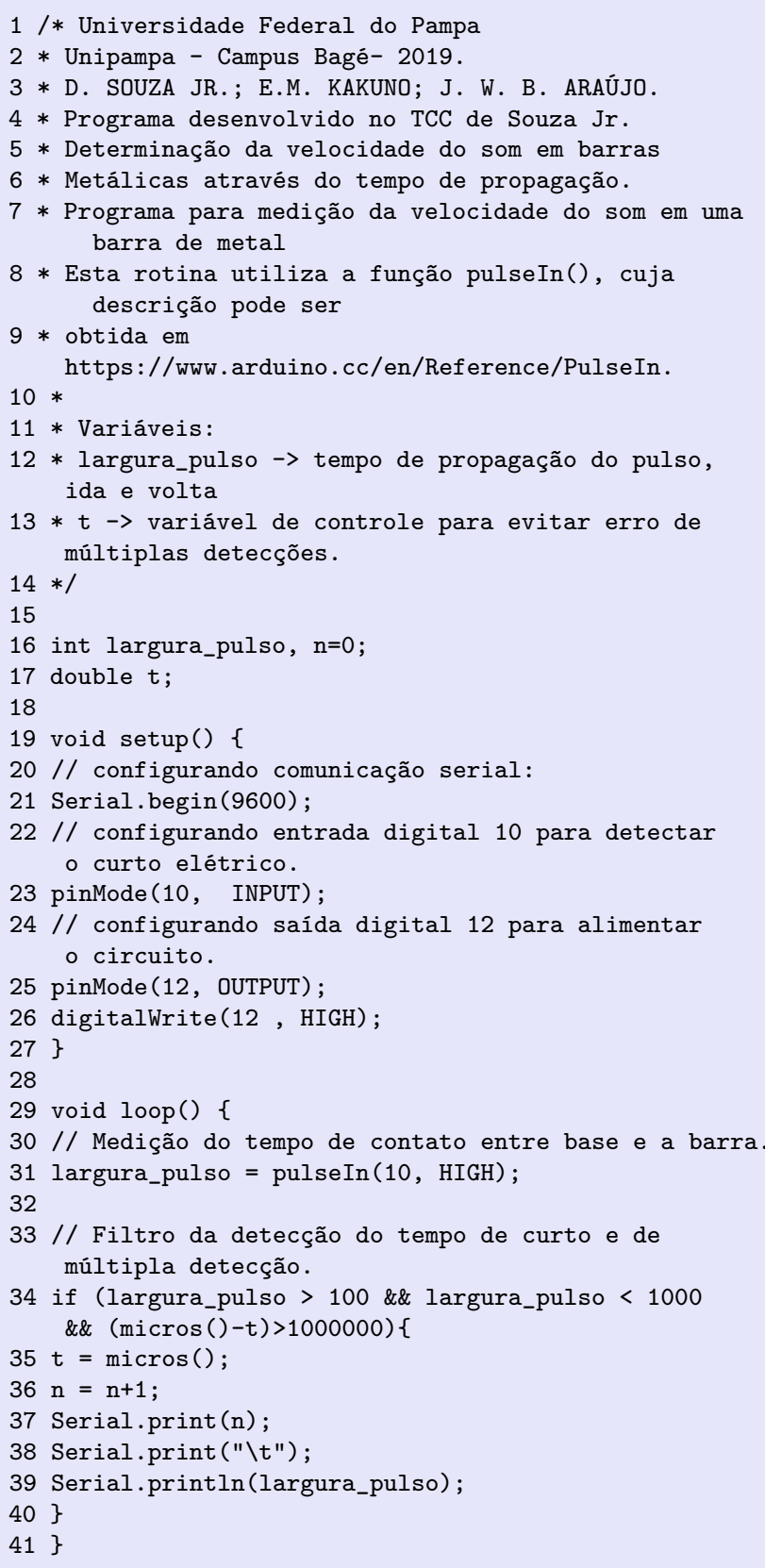

recomendável confirmar o tempo de espera executado pela função, pois em testes prévios verificou-se uma divergência entre o valor programado e o valor executado pela função.

O filtro digital está programado para realizar duas funções durante o processo de medidas, primeira, selecionar pulsos dentro do intervalo de $100 \mathrm{~s}$ a $1000 \mathrm{~s}$, que foi escolhido em função do menor e do maior tempo esperado durante a coleta de dados com as barras, sendo para menor, $050 \mathrm{~cm}$, da ordem de $200 \mathrm{~s}$ e para a maior, 200 $\mathrm{cm}$, da ordem de $800 \mathrm{~s}$.

A segunda finalidade do filtro digital é selecionar apenas o primeiro ponto, correspondendo ao primeiro curto, contato da barra com a base, assim descartando os demais pulsos que ocorrerem no intervalo de um segundo. Ela foi implementada, pois a partir da execução do experimento percebeu-se que somente o primeiro contato fornece pontos sistemáticos.

O programa começa com a declaração das variáveis utilizadas, a "largura_pulso" que armazena o tempo medido, "n" que armazena o número de coletas, e "t" a variável de controle para evitar erro de múltiplas detecções. A função "void setup ()$\{\ldots\}$ " é o espaço destinado a configurações, nela é iniciado a comunicação serial entre o Arduino e o computador, "Serial.begin(9600)" e em seguida são configurados os pinos digitais utilizados, o D10 como entrada digital, "pinMode(10, INPUT)", o D12 como saída digital, "pinMode(12, OUTPUT)", que ainda é configurado para estado alto, "digitalWrite $(12$, $H I G H)$ ".

A função "void loop ()$\{\ldots\}$ " é executada pelo Arduino de forma cíclica, nela chama-se a função pulseIn e salva o valor retornado na variável, "largura_pulso = pulseIn $(10$, $H I G H)$ )". No filtro, "if (largura_pulso >100 \&\&...", o valor salvo na variável "largura_pulso" é comparado com os limites inferior e superior, de 100 e 1000 microssegundos respectivamente. Também é verificado quanto tempo se passou desde do último valor validado pelo filtro, eliminando pulsos subsequentes em até um segundo. Se esses critérios não forem atendidos, o programa volta para a função pulseIn; caso contrário, o valor é válido, e assim atualiza-se o valor da variável de controle temporal, "t $=\operatorname{micros}() "$ e são publicados na serial, "Serial.print()", o número da medida "n", e o valor medido, "largura_pulso".

\section{Resultados}

Durante os testes foram realizados uma série de medidas com uso parcial do filtro, sem habilitar a verificação dos limites de tempo, relacionados aos comprimentos das barras, visto que o intuito foi de qualificar o instrumento. Durante os testes foram utilizadas barras de aço com revestimento em cobre, hastes de aterramento comercial para fins de aterramento elétrico, com comprimento de 050, 100, 150 e $200 \mathrm{~cm}$ e diâmetro de 1,0 cm, uma base 
de aço que corresponde a um segmento de trilho de trem com massa de 15,6 kg.

As barras foram sistematicamente abandonadas (em direção a base) em queda livre, normal à base, às distâncias de $05,10,15,20,25$ e $30 \mathrm{~cm}$. Um conjunto representativo de dados coletados é mostrado na Figura 3, que correspondem a 26 lançamentos da barra de $150 \mathrm{~cm}$ a uma altura de $10 \mathrm{~cm}$.

Observa-se que o intervalo de tempo do pulso se propagar duas vezes na barra (ida e volta) é na ordem de $600 \mathrm{~s}$. Os autores atribuem os três pontos mais dispersos da Figura 3 a erros de execução, como por exemplo a colisão ter ocorrido em uma região não homogênea da base (irregularidade da superfície e/ou oxidação), ou a barra não ter colidido com a base na proximidade de 90 graus. Também o resultado da Figura 3 propicia a discussão junto aos alunos quanto à utilidade de ferramentas estatísticas e de tratamento de dados.

Um quadro com um sumário dos resultados é mostrado na Tabela 1. Detalhes destes testes, inclusive com outros metais (tubo de alumínio e tubo de cobre) estão disponíveis no trabalho de Souza Jr. [6].

Na tabela 1 encontram-se os valores médios e suas incertezas (expressas em dois desvios padrões), calculados para a velocidade do som na barra, através da razão dos comprimentos pelos respectivos tempos médios medidos nas diversas alturas de lançamento. Na periferia da tabela pode-se averiguar os valores médios da velocidade de propagação do pulso e seus respectivos desvios.

Considerando todo o espectro de medidas realizado, barras de $50 \mathrm{~cm}$ a $200 \mathrm{~cm}$ e alturas entre $05 \mathrm{~cm}$ a $30 \mathrm{~cm}$, o resultado médio da velocidade do som da barra foi de $4,7(2) \mathrm{km} / \mathrm{s}$, com uma incerteza de $4,3 \%$. Speziali e Veas reportam para o ferro, material mais próximo ao aço, o valor de $5,3(5) \mathrm{km} / \mathrm{s}$, que corresponde a uma incerteza de $9,4 \%$. As incertezas são da mesma ordem de grandezas,

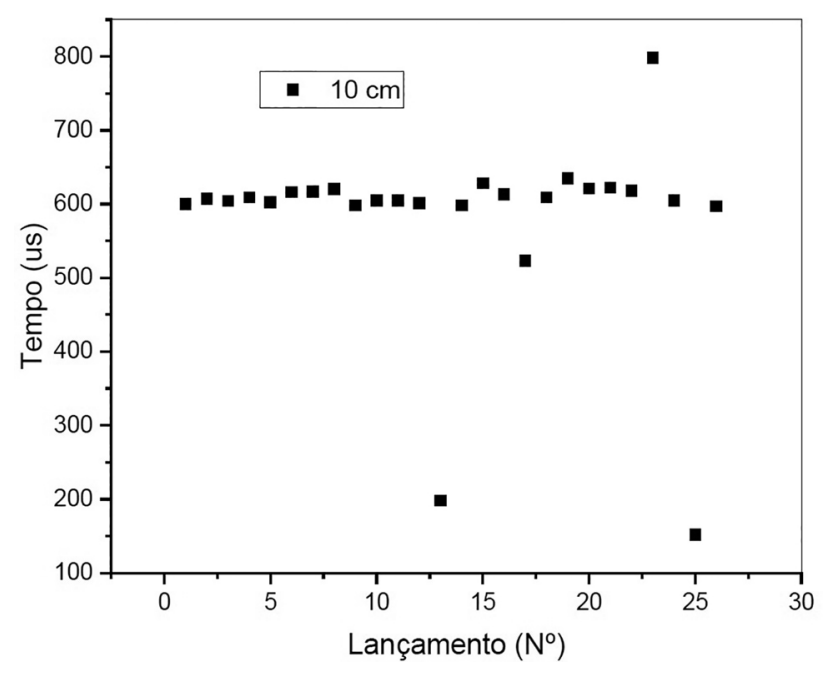

Figura 3: Tempo correspondente da onda sonora propagar em uma barra de aço de $150 \mathrm{~cm}$ de comprimento no trajeto de ida e volta. pois a técnica proposta é a mesma (lançamento da barra sobre um bloco rígido), diferindo somente na forma de medição do tempo.

O objetivo de realizar diversas medidas com diferentes tamanhos de um mesmo material é o de analisar a melhor condição de se reproduzir o experimento, com maior precisão e acurácia. Indica-se uma haste de $100 \mathrm{~cm}$ a $200 \mathrm{~cm}$ e lançamentos entre as alturas de $10 \mathrm{~cm}$ a 25 $\mathrm{cm}$, visto que os dados para estas condições apresentam menores dispersões.

Uma forma de validar o processo de medição é analisar a linearidade do tempo medido em função do comprimento da barra, pois assumindo que a velocidade é constante, modelo de onda em meio homogêneo, espera-se uma relação linear entre o tempo de propagação e o comprimento da barra. A Figura 4 apresenta os resultados de medições dos tempos para as diferentes barras de aço, foram feitos 26 lançamentos com cada barra a altura de $15 \mathrm{~cm}$.

Cada barra gerou no gráfico da Figura 4, um conjunto de pontos na horizontal (no tempo), a largura do conjunto, corresponde a sua dispersão temporal total e cada cor corresponde a um comprimento de barra. Esta mesma analise fora realizada com as demais alturas, e observouse o mesmo comportamento. Nota-se uma linearidade entre o tempo de propagação e o comprimento da barra, dentro da dispersão, isso dá indício de que o método é adequado para a determinação da velocidade do som, para fins didáticos.

Verificou-se ainda a confiabilidade do Arduino na medida do intervalo de tempo, conectando-se um osciloscópio no resistor R1 para observar o pulso elétrico no pino D10, Figura 1. Com a barra de $150 \mathrm{~cm}$, em um lançamento de $15 \mathrm{~cm}$ de altura, encontrou-se $640 \mathrm{~s}$ no osciloscópio e 632 s no Arduino, na Figura 5 tem-se a representação do resultado do osciloscópio, portanto a medição do tempo com Arduino mostra-se confiável.

Foi realizado um arranjo alternativo em que a barra de $200 \mathrm{~cm}$ foi suspensa, na horizontal, em uma de suas extremidades foi instalado uma pastilha (transdutor) piezoelétrico, esta foi conectada na entrada de um osci-

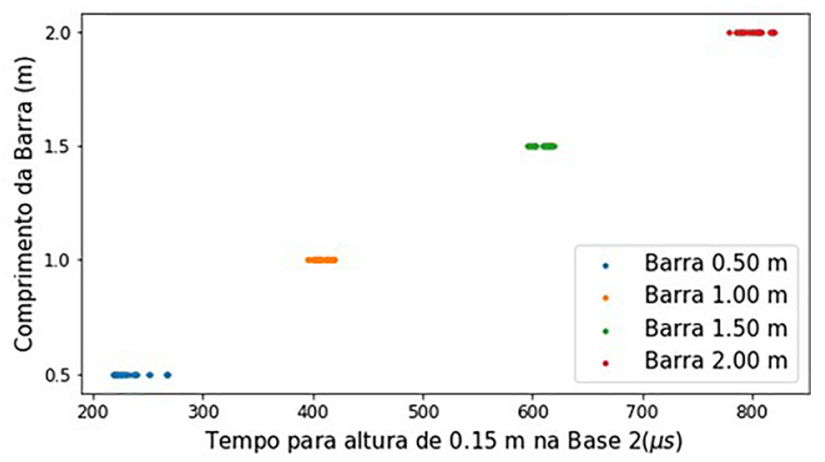

Figura 4: Lineariadade dos tempos de propagação em função do comprimento das barras de aço para altura de $15 \mathrm{~cm}$. 
Tabela 1: Velocidade do som e desvios padrões $(\mathrm{km} / \mathrm{s})$ para barras de aço de diferentes comprimentos, soltas de diferentes alturas $(\mathrm{cm})$.

\begin{tabular}{|l|c|c|c|c|c|}
\hline Altura & Barra 0,5 m & Barra 1,0 m & Barra 1,5 m & Barra 2,0 m & Méd. \\
\hline $\mathbf{5}$ & $4,2(4)$ & $4,4(3)$ & $4,7(2)$ & $4,7(2)$ & $4,5(3)$ \\
\hline $\mathbf{1 0}$ & $4,2(6)$ & $4,6(1)$ & $4,9(1)$ & $4,9(2)$ & $4,6(2)$ \\
\hline $\mathbf{1 5}$ & $4,3(5)$ & $4,7(2)$ & $4,9(1)$ & $5,0(1)$ & $4,7(2)$ \\
\hline $\mathbf{2 0}$ & $4,3(3)$ & $4,7(1)$ & $5,0(1)$ & $5,0(1)$ & $4,7(1)$ \\
\hline $\mathbf{2 5}$ & $4,3(4)$ & $4,8(2)$ & $4,9(2)$ & $5,0(1)$ & $4,7(2)$ \\
\hline $\mathbf{3 0}$ & $4,3(4)$ & $4,8(2)$ & $5,0(3)$ & $5,0(1)$ & $4,8(2)$ \\
\hline Méd. & $4,3(4)$ & $4,7(2)$ & $4,9(2)$ & $4,9(1)$ & $4,7(2)$ \\
\hline
\end{tabular}

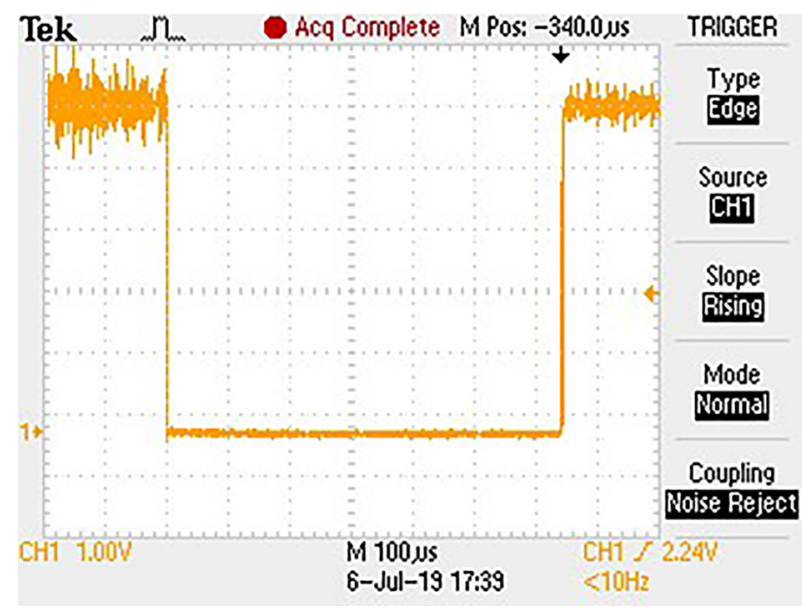

Figura 5: Captura de tela (Trigger) do lançamento da barra de $1,5 \mathrm{~m}$ na base, mostrando o tempo de contato.

loscópio, e gerou-se um pulso na barra batendo em sua outra extremidade com um martelo, Figura 6 .

Identifica-se o intervalo entre os picos, com amplitude acima de $200 \mathrm{mV}$, como o tempo de propagação do pulso na barra (ida e volta), e a amplitude quase constante, dos pulsos, mostra que o tempo de amortecimento é longo, ou seja, pouca energia é "perdida" em reflexões nas extremidades da barra. O intervalo de tempo medido é compatível com o determinado pelo Arduino, da ordem de $800 \mathrm{~s}$ para a barra de $200 \mathrm{~cm}$.

O modelo teórico utilizado para obter a velocidade (v) da onda relaciona o módulo de Young $(Y)$, inverso da elasticidade, com a densidade do material $(\rho)$, conforme Hessel [7].

$$
v=(Y / \rho) 1 / 2
$$

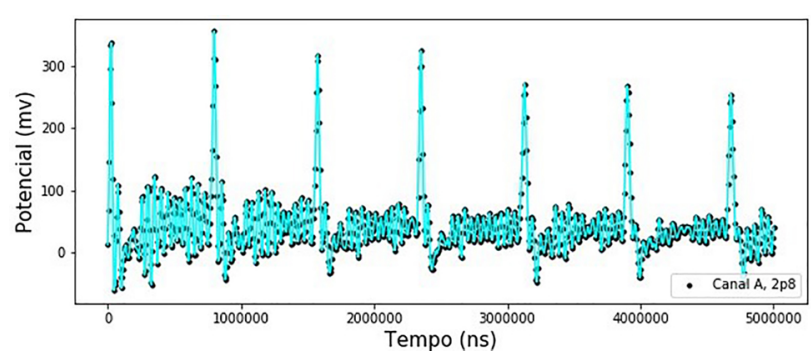

Figura 6: Imagem dos pulsos gerados na haste pelo cristal piezoelétrico.
O tipo de material empregado na barra de aterramento é normalmente o aço 1020 ou 1010 com revestimento de cobre, conforme os fabricantes de hastes de aterramento. E as propriedades dos aços 1020 e 1010 foram obtidas no portal MatWeb [8], o aço 1020 é normalmente constituído por ferro $(99,08-99,53 \%)$, carbono $(0,17-0,23$ $\%$ ), e outras impurezas em menor quantidade, possui densidade volumétrica de $7,87 \times 10^{3} \mathrm{~kg} / \mathrm{m}^{3}$ e módulo de Young de $186 \mathrm{GPa}$. O aço 1010 é normalmente constituído por ferro $(99,18$ - 99,62\%), carbono (0,08 - 0,13 $\%$ ), e outras impurezas em menor quantidade, possui densidade volumétrica de $7,87 \times 10^{3} \mathrm{~kg} / \mathrm{m}^{3}$ e seu módulo de Young varia de 200 a $205 \mathrm{GPa}$. As respectivas velocidades teóricas são de $4.861 \mathrm{~m} / \mathrm{s}$ e entre $5.041 \mathrm{~m} / \mathrm{s}$ e 5.104 $\mathrm{m} / \mathrm{s}$, os resultados obtidos concordam dentro de $3,3 \%$ em relação ao esperado pelo aço 1020 , e associando, em quadratura, esta incerteza com a incerteza aleatória $(4,7$ \%), obtido através da dispersão das medidas, obtém-se uma incerteza total de 5,3\%.

\section{Conclusão}

Considerando todo o espectro de medidas realizado, barras de $50 \mathrm{~cm}$ a $200 \mathrm{~cm}$ e alturas entre $05 \mathrm{~cm}$ a $30 \mathrm{~cm}$, o resultado médio da velocidade do som da barra foi de $4,7(2) \mathrm{km} / \mathrm{s}$, próximo ao valor teórico de $4,861 \mathrm{~km} / \mathrm{s}$ para o aço 1020. Os resultados mostraram ainda que barras de aterramento com comprimento entre 100 e $200 \mathrm{~cm}$, e lançamentos com alturas entre 10 e $25 \mathrm{~cm}$, resultam em condições mais adequadas de reprodução do experimento. Verificou-se a linearidade do tempo de vôo em função do comprimento das barras, a medição do tempo de vôo pelo Arduino com um osciloscópio, a propagação do pulso na barra com um transdutor piezoelétrico com um osciloscópio, e os resultados indicam que a instrumentação proposta é adequada para a determinação da velocidade do som em barras metálicas. Esta instrumentação foi aplicada em uma turma de terceiro ano do ensino médio e se mostrou robusta, uma descrição desta aplicação e seu roteiro encontram-se em Souza Jr. [6]. O autor D. B. S. Jr. agradece ao Programa Residência Pedagógica da Unipampa, subprojeto Física-Química. Portaria CAPES $\mathrm{n}^{\circ} 175$, Agosto de 2018. 


\section{Referências}

[1] N.L. Speziali e F.O. Veas, Revista Brasileira de Ensino de Física 8, 3 (1986).

[2] E. Huggins, The Physics Teacher 46, 13 (2008).

[3] T. Key, R. Smidrovskis e M. From, The Physics Teacher 38, 76 (2000)

[4] M. Se-yuen, N. Yee-kong e W. Kam-wah, Physics Education 35, 439 (2000).

[5] https://www.arduino.cc/en/Reference.PulseIn, acessado em 13/04/2020.

[6] D.B. Souza Jr., E.M. Kakuno e J.W.B. Araújo, Um experimento para determinação da velocidade do som em barras metálicas. Trabalho de Conclusão de Curso, Universidade Federal do Pampa, Bagé (2019).

[7] R. Hessel, A.A. Freschi, E.C. Rosado e L.A. Barreiro, Revista Brasileira de Ensino de Física 38, e2309 (2016).

[8] http://www.matweb.com/, acessado em 13/04/2020. 